\title{
Demonstration of Free Radical Generation in "Stunned" Myocardium of Intact Dogs with the Use of the Spin Trap $\alpha$-Phenyl N-Tert-Butyl Nitrone
}

\author{
Roberto Bolli, Bharat S. Patel, Mohamed O. Jeroudi, Edward K. Lai, ${ }^{\star}$ and Paul B. McCay* \\ Experimental Animal Laboratory, Section of Cardiology, Department of Medicine, Baylor College of Medicine, Houston, Texas 77030, \\ and *Molecular Toxicology Division, Oklahoma Medical Research Foundation, Oklahoma City, Oklahoma 73104
}

\begin{abstract}
Recent studies suggest that oxygen free radicals may mediate postischemic myocardial dysfunction ("stunning"), but all the evidence for this hypothesis is indirect. Thus, we used electron paramagnetic resonance (EPR) spectroscopy and the spin trap, $\alpha$-phenyl $N$-tert-butyl nitrone (PBN), to directly investigate whether free radicals are produced after a 15-min coronary artery occlusion and subsequent reperfusion in 30 open-chest dogs. After intracoronary infusion of PBN, EPR signals characteristic of oxygen- and carbon-centered radical adducts were detected in the venous blood draining from the ischemic/reperfused vascular bed. The myocardial release of $\mathrm{PBN}$ adducts began during coronary occlusion but increased dramatically in the first few minutes after reperfusion. After this initial burst, the production of radicals abated but did not cease, persisting up to $3 \mathrm{~h}$ after reflow. The EPR spectra $\left(a_{\beta}^{\mathrm{H}}=2.67-2.79 \mathrm{G} ; \mathrm{a}_{\mathrm{N}}\right.$ $=14.75-15.00 \mathrm{G}$ ) were consistent with the trapping by PBN of secondary oxygen- and carbon-centered radicals, such as alkoxy and alkyl radicals, which could be formed by reactions of primary oxygen radicals with membrane lipids. There was a linear, direct relationship between the magnitude of PBN adduct production and the degree of ischemic flow reduction. Recovery of contractile function (measured as systolic wall thickening) after reperfusion was greater $(P<0.05)$ in dogs given PBN than in controls.

This study demonstrates that reversible regional myocardial ischemia in the intact animal is associated with prolonged free radical generation, and that the intensity of such generation is related to the severity of ischemia. The results provide direct evidence to support the hypothesis that reactive oxygen metabolites contribute to the persistent contractile dysfunction (myocardial stunning) observed after brief ischemia in vivo.
\end{abstract}

\section{Introduction}

Myocardium reperfused after brief, reversible ischemia exhibits prolonged depression of contractile function $(1,2)$ or "stunning" (3), which is associated with a variety of ultrastructural, biochemical, vascular, and other functional abnormalities (1-6). In recent years, a number of studies have suggested that postischemic myocardial dysfunction may be mediated in part by the generation of such reactive oxygen species as su-

Address reprint requests to Dr. Bolli, Section of Cardiology, Baylor College of Medicine, 6535 Fannin, MS F-905, Houston, TX 77030.

Received for publication 6 October 1987 and in revised form 20 January 1988.

J. Clin. Invest.

(c) The American Society for Clinical Investigation, Inc.

0021-9738/88/08/0476/10 \$2.00

Volume 82, August 1988, 476-485 peroxide anion $\left({ }^{\circ} \mathrm{O}_{2}^{-}\right)$, hydrogen peroxide $\left(\mathrm{H}_{2} \mathrm{O}_{2}\right)$, and hydroxyl radical $\left({ }^{\circ} \mathrm{OH}\right)$. Thus, it has been shown that the recovery of the stunned myocardium is enhanced by agents that either scavenge oxygen metabolites, such as superoxide dismutase and catalase (7-9), $\mathrm{N}$-2-mercaptopropionylglycine (10), and dimethylthiourea (11), or prevent their generation, such as allopurinol (12), oxypurinol (13), and desferrioxamine (14). All the evidence provided by these studies, however, is indirect and, therefore, inconclusive. Clearly, in order to definitively establish a role of oxygen metabolites in postischemic dysfunction, it will be necessary to develop techniques that can directly demonstrate free radical production in the stunned myocardium in the presence and absence of antioxidant interveritions.

Efforts in this direction have recently been made: Zweier et al. (15) have used electron paramagnetic resonance (EPR) ${ }^{1}$ spectroscopy in isolated buffer-perfused rabbit hearts subjected to global ischemia, and have shown that the myocardial concentration of oxygen-centered free radicals increases markedly immediately after reperfusion. Using EPR and spin traps, other investigators (16-18) have also noted production of free radicals in isolated rat hearts undergoing global ischemia and reperfusion. These studies (15-18) have provided direct evidence for free radical production in the postischemic myocardium. However, because of the numerous fundamental differences between the buffer-perfused, nonworking heart subjected to global ischemia in vitro and the blood-perfused, working heart subjected to regional ischemia in the intact animal, results obtained in the former cannot necessarily be extrapolated to the latter. Thus, direct evidence for free radical generation in the stunned myocardium is still lacking.

Although the EPR methods of Zweier et al. (15) have been very useful in isolated hearts, their application to intact animals with regional ischemia would have major practical limitations. First, it is very difficult to freeze-clamp ischemic regions in situ. Myocardial biopsies could be obtained, but the quantity of tissue available would be small and there would be an inevitable delay in achieving the very low temperatures needed $\left(77^{\circ} \mathrm{K}\right)$. Given the highly transient nature of reactive free radicals, even small delays may cause artifacts. Secondly, even if biopsies could be used, it would be difficult to determine the time course of free radical production. This would require either a very large number of biopsies in the same animal or a very large number of animals. Determining the time course of free radical production is not only important from a conceptual standpoint, but also necessary for assessing the effect of a putative scavenger in quantitative terms.

To overcome these problems, we have developed a different approach based on spin-trapping techniques. The rationale

1. Abbreviations used in this paper: EPR, electron paramagnetic resonance; LV, left ventricular; PBN, $\alpha$-phenyl $N$-tert-butyl nitrone. 
for using spin traps was that these agents react with free radicals to form stable adducts that can be detected, identified, and quantitated (19). The primary goal of the present study was to demonstrate production of free radicals in the stunned myocardium in the intact animal with the use of the spin trap $\alpha$-phenyl $N$-tert-butyl nitrone (PBN). Additional objectives were $(a)$ to determine the time course of free radical generation, $(b)$ to define the relation between severity of ischemia and intensity of free radical formation, and $(c)$ to develop a model that allows quantitative assessment of spin adduct production by the ischemic/reperfused myocardium.

\section{Methods}

\section{Experimental preparation}

This study was performed using the experimental model of postischemic dysfunction in which antioxidant interventions were previously shown to be beneficial $(7,10-12,14)$, namely, a 15-min coronary artery occlusion followed by reperfusion. The techniques employed have been described in detail previously $(7,10-12,14,20,21)$. Briefly, pentobarbital-anesthetized dogs of either sex (17-36 kg) were instrumented with a snare around either the posterior descending or the obtuse marginal coronary artery, a Doppler flow velocity probe around the vessel distal to the snare, an epicardial Doppler wall thickening probe (20) on the region to be rendered ischemic and another on the anterior (control) left ventricular (LV) wall, polyethylene catheters in the aorta and left atrium, and a No. 6F Millar pressure transducer (Millar Instruments, Inc., Houston, TX) in the left ventricle through a stab wound in the anterior wall. A 27-gauge needle connected to a lymphangiographic catheter was introduced into the artery to be occluded, just distal to the snare, for infusion of PBN or vehicle. The site of occlusion was carefully selected to ensure that no branches were present between the snare and the needle (so that the entire occluded/ reperfused vascular bed received PBN) or between the needle and the Doppler flow probe. To prevent clotting, heparin was given immediately after insertion of the needle (3,000 $\mathrm{U}$ i.v.) and continuously thereafter $(500 \mathrm{U} / \mathrm{h})$. A No. $8 \mathrm{~F}$ Sones catheter (USCI Division, C. $\mathbf{R}$. Bard, Inc., Billerica, MA) was introduced into the coronary sinus and advanced into the vein which accompanied the artery to be occluded (either the posterior interventricular or the left marginal vein). In its final position, the tip of the catheter was at least $0.5 \mathrm{~cm}$ distal to the intracoronary needle, so that any contamination of blood samples with venous effluent from other vascular beds was minimized.

Particular care was taken to ensure that fundamental physiologic parameters were within normal limits for the entire duration of the protocol. Only dogs with hematocrit $>30 \%$ were admitted to the study. Supplements of potassium chloride (10-20 meq) were given i.v. to maintain plasma potassium concentration in the range of 4.0-5.0 meq/liter. The rate of fluid replacement was standardized at $80 \mathrm{ml}$ of normal saline per hour. Throughout the study, body temperature was kept within physiological limits by adjusting a heating blanket, while arterial $\mathrm{pH}$ and $\mathrm{PO}_{2}$ were maintained in the normal range by adjusting the ventilatory parameters (14). Dogs in which arterial $\mathrm{PO}_{2}$ was $<60$ $\mathrm{mmHg}$ or arterial $\mathrm{pH}<7.36$ in spite of these adjustments were not used.

\section{Experimental protocol}

The selected coronary artery was occluded for $15 \mathrm{~min}$ and then reperfused for $3 \mathrm{~h}$. This duration of ischemia was selected because it is well established that it does not result in myocardial necrosis in the dog (22), but nevertheless it does produce prolonged depression of contractility $(1-3,21)$. Regional myocardial blood flow to the occluded and nonoccluded vascular beds was determined by the radioactive microspheres technique (14) 10 min before occlusion (before PBN administration), $10 \mathrm{~min}$ after occlusion, and $60 \mathrm{~min}$ after reperfusion. Animals that developed ventricular fibrillation were excluded. After killing the dogs, the size of the occluded/reperfused coronary vascular bed was determined by a postmortem dual-perfusion technique (14). Absence of irreversible tissue damage was confirmed in all animals by incubating transverse slices of the heart in $1 \%$ triphenyltetrazolium chloride (23).

The spin-trapping agent, PBN, was administered directly into the selected coronary artery. This agent was chosen because $(a)$ it is known to form relatively long-lived adducts with oxygen- and carbon-centered radicals (19), (b) it has been shown to be compatible with normal myocardial function at low concentrations (24), and $(c)$ it has been previously established that it can function as an effective free radical trapping agent in vivo (25-28). PBN (Sigma Chemical Co., St. Louis, MO) was dissolved at a concentration of $5 \mathrm{mg} / \mathrm{ml}$ in a mixture of $80 \%$ normal saline and $20 \%$ water, so that the infusate was isosmotic with the plasma (osmolality $=284 \mathrm{mosmol} / \mathrm{kg}$ ). Because flow in the artery to be occluded varied from dog to dog, the rate of $\mathrm{PBN}$ infusion (in milligrams per minute) was normalized to flow (in milliliters per minute), so as to achieve the same concentration of PBN in the coronary arterial blood in all dogs. Consequently, the dose of PBN can be expressed as $(\mathrm{mg} / \mathrm{min}) /(\mathrm{ml} / \mathrm{min})$ or $\mathrm{mg} / \mathrm{ml}$, which is the calculated PBN concentration in the coronary arterial blood. The volume of solution infused (which ranged from 0.4 to $3.4 \mathrm{ml} / \mathrm{min}$ ) was equal to $6 \%$ of coronary blood flow in all dogs. Before and after PBN administration, the artery was infused with an equivalent volume of vehicle. Dogs ( $n$ $=38$ ) were assigned to the following groups:

Group I (radical trapping during ischemia and reperfusion). In these animals $(n=5)$, PBN was infused to achieve a coronary arterial concentration of $285 \mu \mathrm{g} / \mathrm{ml}(1.6 \mathrm{mM})$ beginning $5 \mathrm{~min}$ before coronary occlusion and ending $10 \mathrm{~min}$ after reperfusion. Baseline coronary blood flow ranged from 7.9 to $33.3 \mathrm{ml} / \mathrm{min}$; the rate of infusion varied accordingly from 2.3 to $9.5 \mathrm{mg} / \mathrm{min}$ (mean: $4.4 \mathrm{mg} / \mathrm{min}$ ). During coronary occlusion, when no Doppler flow signal was available, the rate of infusion was decreased to $10 \%$ of preocclusion to take into account the fact that collateral flow in open-chest dogs averages $10-15 \%$ of preocclusion $(7-12,14)$ (higher infusion rates would have resulted in significant increases in PBN concentration). The preocclusion rate of PBN infusion was restarted $20 \mathrm{~s}$ before reperfusion.

Group II (radical trapping beginning at reperfusion). In these dogs $(n=5), \mathrm{PBN}$ was infused at the same rate as in the previous group, but beginning $20 \mathrm{~s}$ prior to reperfusion and ending $10 \mathrm{~min}$ thereafter. As in group I, the rate of PBN infusion was selected on the basis of the preocclusion measurement of coronary blood flow.

Group III (radical trapping beginning after reperfusion). PBN was infused for $10 \mathrm{~min}$ at the same rate as in groups I and II, but beginning at $30 \mathrm{~min}(n=3), 1 \mathrm{~h}(n=3)$, or $2 \mathrm{~h}(n=3)$ after reperfusion.

Group IV (control group for EPR signals). In these animals $(n=11)$, PBN was infused at the same rate as in groups I, II, and III, but no coronary occlusion was performed. Because the longest duration of infusion in the experimental groups was $30 \mathrm{~min}$ (group I), in these dogs the administration of PBN was limited to $40 \mathrm{~min}$. Radioactive microspheres were injected before infusion of PBN and $40 \mathrm{~min}$ after start of infusion. This control group was necessary to ensure that the EPR signals observed in groups I, II, and III were not the result of nonspecific actions of PBN unrelated to ischemia and reperfusion.

Group V (control group for recovery of function). These dogs $(n=8)$ underwent the same experimental protocol as group I and II except for the fact that PBN was not administered (equivalent volumes of vehicle were infused through the intracoronary needle). The reason for studying this control group was to determine whether the infusion of PBN in group I and II produced any beneficial effect on the recovery of contractile function in the stunned myocardium.

\section{EPR analysis}

By means of syringes containing $100 \mathrm{U}$ of heparin, blood samples $(6$ $\mathrm{ml}$ ) were drawn over a 60 -s period from the aorta or from the appropriate coronary vein and immediately centrifuged to separate the plasma. The plasma specimens were frozen at $-70^{\circ} \mathrm{C}$ for subsequent analysis by EPR spectroscopy. The technique used to detect spin adducts of reactive free radicals in vivo has been previously described in 
detail (25-28). Briefly, total lipids were extracted from $3.0 \mathrm{ml}$ of each plasma sample by adding $15 \mathrm{vol}$ of chloroform/methanol (2:1 vol/vol). To this single-phase system, one-fifth volume of $0.5 \% \mathrm{NaCl}$ was added to bring about phase partitioning in a separatory funnel. The chloroform layer was then recovered and evaporated to dryness. The lipid residue (which would include any radical adducts of PBN) was taken up in $50 \mu$ l of chloroform, transferred to a Pasteur pipette (sealed at the capillary end), and centrifugally forced to the bottom of the capillary end. The pipette was then placed in the sample cavity of an IBMBruker ESP 300 EPR Spectrometer (Karlsruhe, FRG) and scanned for the presence of spin adducts. The spectrometer settings were as follows: microwave power, $19.7 \mathrm{~mW}$; modulation amplitude, $1 \mathrm{G}$; time constant, $1.25 \mathrm{~s}$; scan range, $100 \mathrm{G}$; and scan time, $8 \mathrm{~min}$. All spectra were recorded at room temperature $\left(25^{\circ} \mathrm{C}\right)$.

\section{Quantification of myocardial release of $P B N$ adducts}

The intensity of the EPR signal (which is proportional to the concentration of spin adducts in the sample) is expressed in arbitrary units; these were obtained by measuring (in millimeters) the height of the first line of the second doublet of the spectrum, adjusted for the gain of the scan (Fig. 1). As indicated in Results, no adducts were detected in the arterial blood samples. Consequently, myocardial release of spin adducts was calculated (in arbitrary units [U] per minute) by multiplying the signal intensity in the plasma of the venous effluent blood (which is proportional to the concentration of the adduct in that blood [U/ml]) by the simultaneous coronary arterial blood flow ( $\mathrm{ml} / \mathrm{min})$; this quantity was normalized to the size of the occluded/reperfused bed (measured in grams). Thus, myocardial release of spin adducts is expressed in arbitrary units per minute per gram of myocardium ( $U / \mathrm{min}$ per $\mathrm{g}$ ). During coronary occlusion, when no Doppler flow signal was available, microsphere flow measurements in milliliters per minute per gram $(\mathrm{ml} / \mathrm{min}$ per $\mathrm{g}$ ) were used.

The total myocardial production of PBN adducts over a given interval of time was calculated by integrating the data with a computerized program. Briefly, the individual values of adduct release at each time point were entered in the computer; from these values, the time course of adduct release for each dog was derived and the area under the curve for a selected time interval was determined.

\section{Assessment of regional myocardial function}

Regional myocardial function was assessed using a pulsed Doppler epicardial wall thickening probe, as previously described $(7,10-12,14$, 21). Theoretical and experimental validation of this technique has been published elsewhere $(20,29)$. The beginning and end of systole were determined from the onset of the rapid upstroke of the LV pressure tracing and the peak negative $\mathrm{d} P / \mathrm{d} t$, respectively (30). Percent systolic thickening fraction was calculated as the ratio of net systolic thickening to end-diastolic wall thickness, multiplied by 100 (20). Net systolic thickening was defined as the maximal systolic increase in wall thickness from the end-diastolic value (30). When paradoxical wall thinning persisted for $50 \%$ or more of systole, the maximal extent of wall thinning was subtracted from wall thickening to give net systolic thickening $(7,10-12,14,21,30)$. At least five beats were averaged at each time point. Because wall thickening can vary markedly with the phase of the respiratory cycle, all measurements were obtained at end expiration.

\section{Statistical analysis}

All values are reported as mean \pm SEM. Analysis of variance was used to compare means among multiple groups and to analyze intragroup variation. This procedure avoids the error inherent in performing multiple pairwise comparisons (31). The correlation between myocardial production of PBN adducts and collateral blood flow was assessed by linear regression analysis using the least-squares method.

\section{Results}

Group I $(n=5)$ : radical trapping during ischemia and reperfusion. Table I summarizes the measurements of hemodynamic

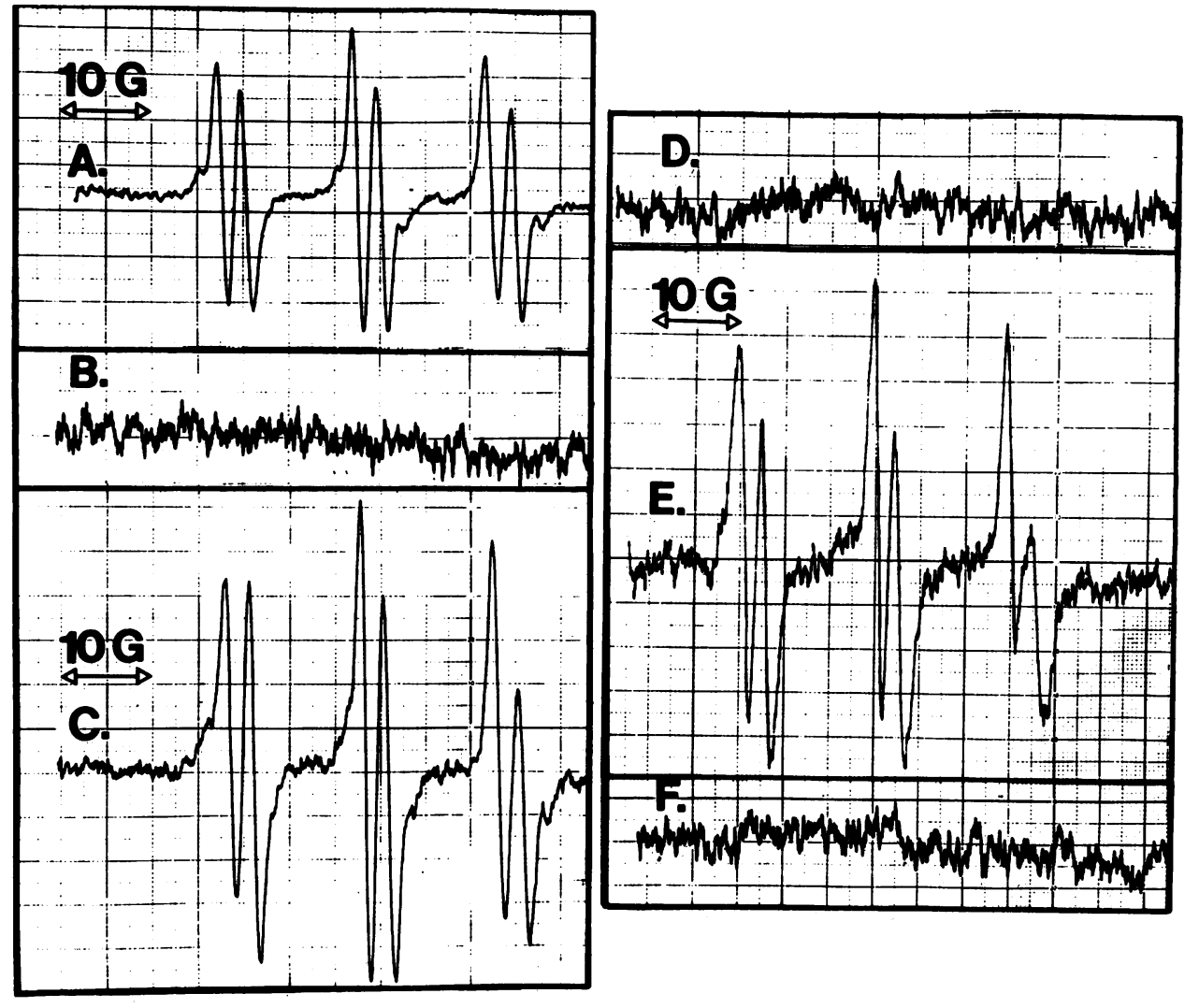

Figure 1. Representative EPR spectra of PBN radical adducts detected in the coronary venous effluent blood. Shown in this figure are signals from plasma samples obtained: $(A) 3$ min after reperfusion in a dog from group I (PBN infusion started 5 min before ischemia) $\left(a_{\mathrm{N}}=14.75, a_{\beta}^{\mathrm{H}}=2.69 \mathrm{G}\right.$; gain, $\left.5 \times 10^{5}\right) ;(B)$ at corresponding time after start of PBN infusion in a control dog (gain, 1 $\left.\times 10^{6}\right)$; $(C) 5$ min after reperfusion in a dog in group II (PBN infusion started $20 \mathrm{~s}$ before reperfusion) $\left(a_{\mathrm{N}}=14.77, a_{\beta}^{\mathrm{H}}=2.69 \mathrm{G}\right.$; gain, $\left.5 \times 10^{5}\right)$; $(D)$ at corresponding time after start of $P B N$ in a second control dog (gain, 1 $\left.\times 10^{6}\right)$; (E) 35 min after reperfusion in a dog in group III (PBN infusion started $30 \mathrm{~min}$ after reperfusion) $\left(a_{\mathrm{N}}=15.00, a_{\beta}^{\mathrm{H}}=2.78\right.$ G; gain, $\left.2 \times 10^{6}\right) ;(F)$ at corresponding time after start of PBN in a third control dog (gain, 1 $\times 10^{6}$ ). 
Table I. Hemodynamic Variables, Wall Thickening in the Nonischemic Zone, Regional Myocardial Blood Flow, and Occluded Bed Size in Groups I, II, and $V^{*}$

\begin{tabular}{|c|c|c|c|c|c|c|}
\hline & \multirow[b]{2}{*}{ Baseline } & \multirow[b]{2}{*}{$\begin{array}{c}\text { 10-min } \\
\text { occlusion }\end{array}$} & \multicolumn{4}{|c|}{ Reperfusion (min) } \\
\hline & & & 30 & 60 & 120 & 180 \\
\hline \multicolumn{7}{|c|}{ Heart rate (beats/min) } \\
\hline Group I & $147 \pm 6$ & $150 \pm 6$ & $151 \pm 7$ & $144 \pm 12$ & $150 \pm 15$ & $154 \pm 16$ \\
\hline Group II & $142 \pm 13$ & $143 \pm 12$ & $141 \pm 14$ & $153 \pm 9$ & $144 \pm 15$ & $150 \pm 12$ \\
\hline Group V & $152 \pm 3$ & $154 \pm 8$ & $156 \pm 4$ & $160 \pm 5$ & $160 \pm 4$ & $163 \pm 6$ \\
\hline \multicolumn{7}{|c|}{ Mean arterial pressure $(\mathrm{mmHg})$} \\
\hline Group I & $105 \pm 9$ & $105 \pm 12$ & $111 \pm 15$ & $118 \pm 14$ & $113 \pm 17$ & $111 \pm 14$ \\
\hline Group II & $118 \pm 11$ & $113 \pm 10$ & $114 \pm 10$ & $120 \pm 8$ & $112 \pm 4$ & $108 \pm 7$ \\
\hline Group V & $117 \pm 8$ & $114 \pm 8$ & $116 \pm 7$ & $113 \pm 7$ & $113 \pm 7$ & $109 \pm 8$ \\
\hline \multicolumn{7}{|c|}{ Mean left atrial pressure $(\mathrm{mmHg})$} \\
\hline Group I & $5 \pm 1$ & $5 \pm 1$ & $4 \pm 1$ & $4 \pm 1$ & $4 \pm 1$ & $4 \pm 1$ \\
\hline Group II & $4 \pm 1$ & $4 \pm 1$ & $4 \pm 1$ & $3 \pm 1$ & $3 \pm 1$ & $4 \pm 1$ \\
\hline Group V & $4 \pm 1$ & $5 \pm 1$ & $4 \pm 1$ & $5 \pm 1$ & $5 \pm 1$ & $5 \pm 1$ \\
\hline \multicolumn{7}{|c|}{ Coronary blood flow $(\mathrm{ml} / \mathrm{min})^{\ddagger}$} \\
\hline Group I & $16 \pm 5$ & 0 & $16 \pm 4$ & $13 \pm 4$ & $14 \pm 3$ & $15 \pm 3$ \\
\hline Group II & $18 \pm 3$ & 0 & $16 \pm 3$ & $17 \pm 3$ & $16 \pm 3$ & $16 \pm 3$ \\
\hline Group V & $20 \pm 3$ & 0 & $16 \pm 1$ & $16 \pm 1$ & $17 \pm 1$ & $16 \pm 1$ \\
\hline \multicolumn{7}{|c|}{$\mathrm{LV} \mathrm{d} P / \mathrm{d} t_{\max }(m m H g / s)^{s}$} \\
\hline Group I & $2,260 \pm 260$ & $2,384 \pm 220$ & $2,329 \pm 225$ & $1,917 \pm 134$ & $1,829 \pm 271$ & $1,851 \pm 288$ \\
\hline Group II & $1,967 \pm 174$ & $2,036 \pm 186$ & $2,008 \pm 121$ & $1,813 \pm 181$ & $1,779 \pm 275$ & $1,790 \pm 271$ \\
\hline Group V & $2,199 \pm 289$ & $1,979 \pm 202$ & $2,007 \pm 187$ & $2,172 \pm 190$ & $2,722 \pm 220$ & $2,034 \pm 211$ \\
\hline \multicolumn{7}{|c|}{ 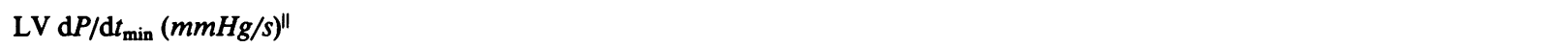 } \\
\hline Group I & $2,741 \pm 456$ & $2,604 \pm 389$ & $2,810 \pm 440$ & $2,567 \pm 365$ & $2,512 \pm 491$ & $2,610 \pm 392$ \\
\hline Group II & $2,494 \pm 283$ & $2,244 \pm 327$ & $2,535 \pm 138$ & $2,192 \pm 286$ & $2,467 \pm 137$ & $2,512 \pm 148$ \\
\hline Group V & $2,887 \pm 489$ & $2,611 \pm 273$ & $2,749 \pm 318$ & $2,952 \pm 299$ & $2,776 \pm 221$ & $2,804 \pm 291$ \\
\hline \multicolumn{7}{|c|}{ Thickening fraction (\%) (NIZ) } \\
\hline Group I & $13 \pm 2$ & $14 \pm 2$ & $12 \pm 3$ & $12 \pm 5$ & $13 \pm 4$ & $12 \pm 4$ \\
\hline Group II & $20 \pm 3$ & $24 \pm 4$ & $23 \pm 7$ & $23 \pm 3$ & $21 \pm 5$ & $20 \pm 5$ \\
\hline Group V & $16 \pm 2$ & $18 \pm 3$ & $18 \pm 2$ & $16 \pm 3$ & $16 \pm 3$ & $15 \pm 3$ \\
\hline \multicolumn{7}{|c|}{ Transmural regional myocardial blood flow $(\mathrm{ml} / \mathrm{min}$ per $\mathrm{g})(\mathrm{IZ})^{* *}$} \\
\hline Group I & $0.94 \pm 0.10$ & $0.24 \pm 0.11$ & - & $0.99 \pm 0.04$ & - & - \\
\hline Group II & $1.18 \pm 0.23$ & $0.27 \pm 0.09$ & - & $0.97 \pm 0.08$ & - & - \\
\hline Group V & $1.02 \pm 0.10$ & $0.22 \pm 0.03$ & - & $0.98 \pm 0.11$ & - & - \\
\hline \multicolumn{7}{|c|}{ Transmural regional myocardial blood flow ( $\mathrm{ml} / \mathrm{min}$ per $\mathrm{g}$ ) (NIZ) } \\
\hline Group I & $1.13 \pm 0.17$ & $1.29 \pm 0.20$ & - & $1.29 \pm 0.05$ & - & - \\
\hline Group II & $1.34 \pm 0.20$ & $1.53 \pm 0.29$ & - & $1.23 \pm 0.13$ & - & - \\
\hline Group V & $1.12 \pm 0.12$ & $1.40 \pm 0.22$ & - & $1.05 \pm 0.12$ & - & - \\
\hline \multicolumn{7}{|c|}{ Occluded vascular bed $(g)$} \\
\hline Group I & \multicolumn{6}{|c|}{$21.9 \pm 3.2 \mathrm{~g}(29.8 \pm 5.2 \%$ of $\mathrm{LV}$ weight $)$} \\
\hline Group II & \multicolumn{6}{|c|}{$24.8 \pm 2.9 \mathrm{~g}(28.2 \pm 3.3 \%$ of $\mathrm{LV}$ weight $)$} \\
\hline Group V & \multicolumn{6}{|c|}{$23.8 \pm 2.4 \mathrm{~g}(30.3 \pm 4.1 \%$ of $\mathrm{LV}$ weight $)$} \\
\hline
\end{tabular}

Data are mean \pm SEM. * In group I the infusion of PBN was started before ischemia and continued until 10 min after reperfusion; in group II the infusion of PBN was started at reperfusion and continued for $10 \mathrm{~min}$; in group V no PBN was given. ${ }^{\ddagger}$ Measured in the artery infused with PBN. ${ }^{5} \mathrm{LV} \mathrm{d} P / \mathrm{d} t_{\max }$, maximal rate of left ventricular pressure rise. " $\mathrm{LV} \mathrm{d} P / \mathrm{d} t_{\min }$, maximal rate of left ventricular pressure fall. ' $\mathrm{NIZ}$, nonischemic zone. ${ }^{* *} \mathrm{IZ}$, ischemic/reperfused zone.

variables, myocardial blood flow, occluded bed size, and LV wall thickening in the nonischemic region for this group as well as groups II and V. The measurements of wall thickening in the ischemic/reperfused region are illustrated in Fig. 2.
Fig. 1 shows representative examples of the EPR signals observed, whereas Fig. 3 summarizes the time course of release of PBN adducts from the ischemic/reperfused region in groups I and II. No spin adducts were detected in the venous effluent 


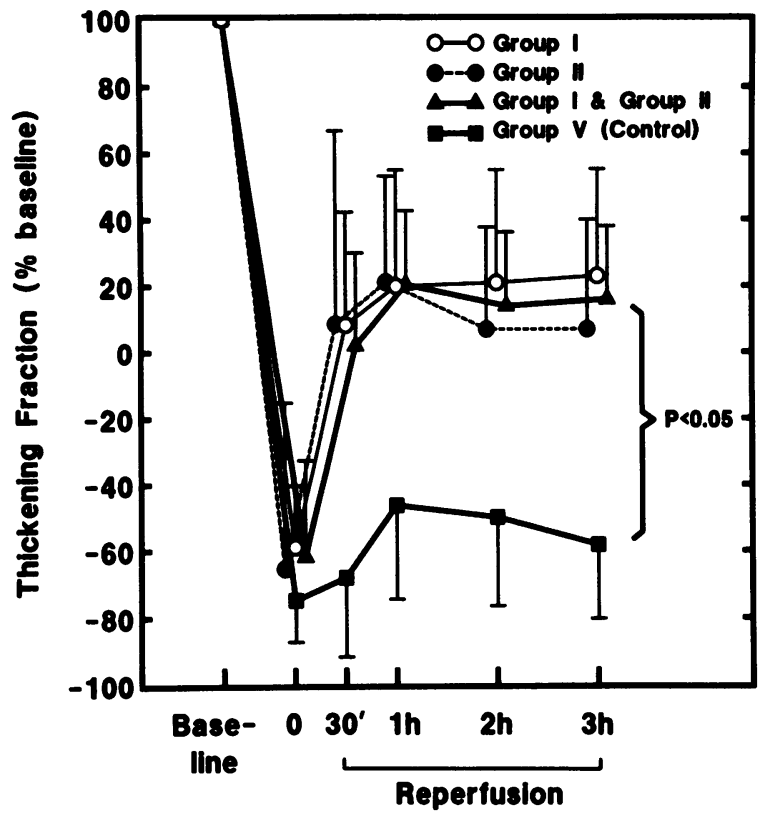

Figure 2. Systolic thickening fraction in the ischemic/reperfused region 5 min after coronary occlusion $(0)$ and at selected times after reperfusion in the following groups: group V (no PBN given, $n=8$ [匹]), group I (PBN infusion started before ischemia, $n=5$ [0], group II (PBN infusion started at reperfusion, $n=5[\bullet]$ ), and groups I and II combined ( $\Lambda$ ). Thickening fraction is expressed as percentage of baseline values. Data are mean \pm SEM. Because of the small number of observations in group I and II, these groups were combined to allow the effect of PBN to be better assessed using a larger sample size. Analysis of variance showed that thickening fraction improved significantly $(P<0.005)$ over time in both group I and group II. In contrast, there was no demonstrable change in thickening fraction during the occlusion and the reperfusion phase in control dogs (group V) $(P=0.31)$. Furthermore, as demonstrated by the bracket, there was a significant $(P<0.05)$ overall difference in thickening fraction after reperfusion between the pooled PBN group and the control group (analysis of variance), indicating that $\mathrm{PBN}$ modified the global pattern of postischemic recovery. before coronary occlusion. An EPR signal characteristic of a radical adduct of PBN appeared in the coronary effluent after occlusion, but the intensity of the signal was weak (average myocardial release $=0.30 \pm 0.30 \mathrm{U} / \mathrm{min}$ per $\mathrm{g}$ at $5 \mathrm{~min}$ and $1.30 \pm 0.80 \mathrm{U} / \mathrm{min}$ per $\mathrm{g}$ at $13 \mathrm{~min}$ ) (Fig. 3). Upon reperfusion, however, there was an immediate, dramatic increase in spin adduct release (Figs. $1 \mathrm{~A}$ and 3). During the first $60 \mathrm{~s}$ of reflow, the amount of adduct drained by the venous system $(75.9 \pm 20.0 \mathrm{U} / \mathrm{min}$ per $\mathrm{g})$ was almost 60 times greater than at $13 \mathrm{~min}$ of ischemia. The release of PBN adducts peaked at 2 min after restoration of blood flow, when it averaged $118.1 \pm 41.2 \mathrm{U} / \mathrm{min}$ per $\mathrm{g}$ (a value 90 times greater than at 13 min of ischemia) (Fig. 3). The release of the trapped radicals declined markedly over the ensuing 20 min but continued (albeit much less intensely) up to $3 \mathrm{~h}$ after reperfusion (Fig. 3). In this group, as well as in groups II and III, no EPR signal was observed in any of the arterial blood samples, which were obtained 5 and 13 min into the occlusion phase and 2, 5, 20, 60 , and $180 \mathrm{~min}$ into the reperfusion phase. Thus, the presence of an EPR signal in the venous effluent blood cannot be ascribed to recirculation of the PBN adducts.

Group II $(n=5)$ : radical trapping beginning at reperfusion. In these dogs the infusion of PBN was started $20 \mathrm{~s}$ before reperfusion and continued for the ensuing $10 \mathrm{~min}$. Hemodynamic variables, wall thickening in the ischemic region, size of occluded bed, and collateral flow were similar to group I (Table I, Fig. 2). As expected, no EPR signal was detected during the occlusion phase. After reperfusion, however, there was marked production of PBN adducts (Fig. $1 C$ ), the magnitude and time course of which were comparable to those observed in group I (Fig. 3). These findings demonstrate that the abrupt increase in the release of PBN adducts observed after reflow in group I does, indeed, represent free radical production by the reperfused myocardium, and is not primarily due to "wash out" of spin trapped radicals accumulated during ischemia.

Relation of adduct production to severity of ischemia. The myocardial production of PBN radical adducts exhibited a

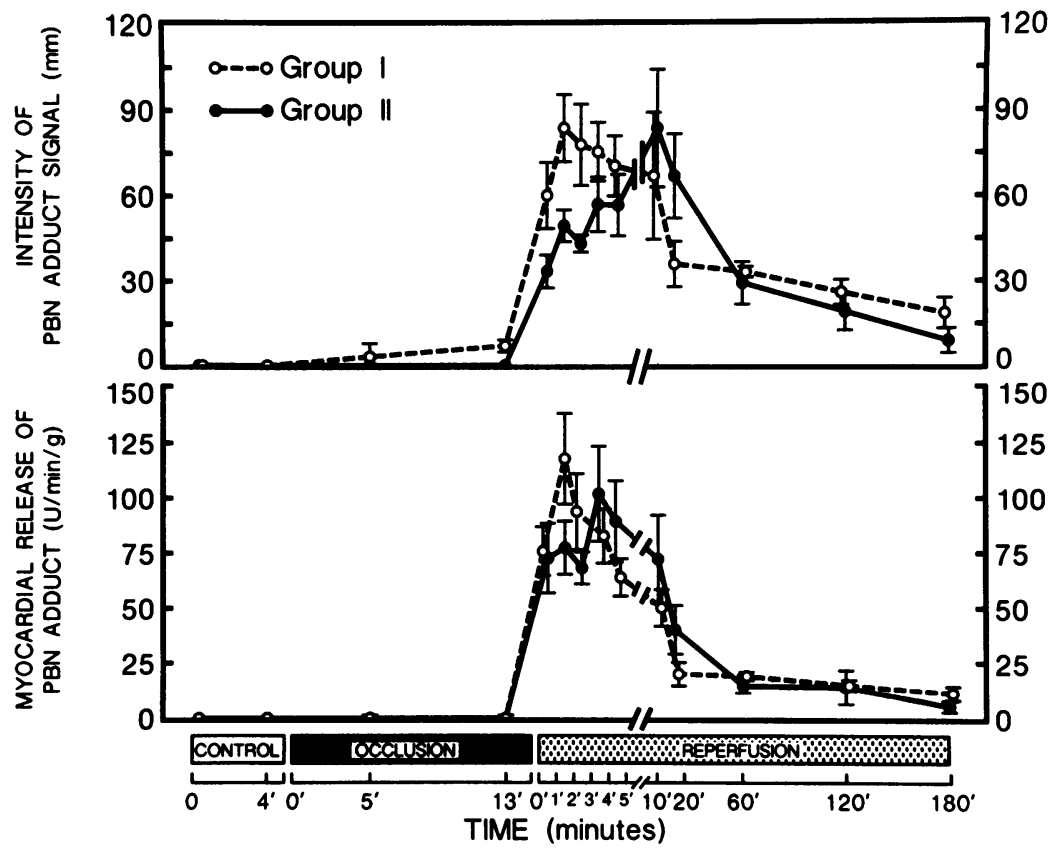

Figure 3. (Upper panel) Intensity of the EPR signals detected in the coronary venous effluent blood in groups I and II. (Lower panel) Time course of myocardial release of PBN adducts in groups I and II. Data are mean \pm SEM. See text for explanation of units used. 
marked individual variability (Fig. 4). Indeed, the total cumulative adduct release (area under the curve) during the $3 \mathrm{~h}$ of reperfusion averaged $4,351 \mathrm{U} / \mathrm{min}$ per $\mathrm{g}$ but ranged from 720 to $9,250 \mathrm{U} / \mathrm{min}$ per $\mathrm{g}$ (a 13-fold difference). In both group I and II, there was a linear and inverse relationship between myocardial production of $\mathrm{PBN}$ adducts after coronary reperfusion and collateral flow to the ischemic region during occlusion. For example, the total amount of spin adducts released during the first 5 min of reperfusion (the critical interval during which the burst of free radical generation took place) correlated with collateral flow in group I $(r=-0.71, n=5)$, group II $(r=-0.91, n=5)$, and groups I and II combined $(r=-0.77$, $n=10$ ) (Fig. 4). Similar results were obtained when collateral flow was related to the total release of adducts observed during the initial $10 \mathrm{~min}$ of reflow (Fig. 4) or during longer periods (for example, for group I and II combined, the $r$ values were -0.69 after $20 \mathrm{~min}$ of reperfusion, -0.73 after $60 \mathrm{~min}$, and -0.75 after $120 \mathrm{~min})$. These data indicate that both the initial burst and the overall intensity of free radical generation after reperfusion are highly variable, and that both are determined by the severity of the antecedent ischemic insult: the greater the degree of hypoperfusion, the greater the production of radicals. Interestingly, the regression lines intercept the $x$-axis at values of collateral flow of $35-40 \%$ of nonischemic zone flow (Fig. 4). Hence, a reduction in blood flow of at least 60-65\% appears to be necessary to trigger the generation of the radical species trapped by PBN.

Group III $(n=9)$ : radical trapping beginning after reperfusion. In these dogs the infusion of PBN was started at various times ( $30 \mathrm{~min}, 1 \mathrm{~h}$, or $2 \mathrm{~h}$ ) after reperfusion and continued for the subsequent $10 \mathrm{~min}$. The animals were similar to groups I and II with respect to the variables reported in Table I (data not shown for the sake of brevity). As expected, no EPR signal was observed until PBN was administered. Immediately after start of PBN infusion a radical adduct appeared in the venous effluent, even when the spin trap was given as late as $2 \mathrm{~h}$ after reperfusion (Fig. $1 E$ shows an EPR signal detected when PBN was begun $30 \mathrm{~min}$ after reperfusion). However, the magnitude of adduct release was relatively small and was similar to that observed in groups I and II at corresponding times after reflow (30 min, $1 \mathrm{~h}$, or $2 \mathrm{~h}$ ). For example, when PBN infusion was started $60 \mathrm{~min}$ after reperfusion, the peak release of radicals (observed at $65 \mathrm{~min}$ ) averaged $21.4 \pm 10.7 \mathrm{U} / \mathrm{min}$ per $\mathrm{g}$; when the infusion was begun $120 \mathrm{~min}$ after reperfusion, the peak release (at $125 \mathrm{~min}$ ) averaged $8.5 \pm 4.9 \mathrm{U} / \mathrm{min}$ per g. These results demonstrate that the prolonged release of $\mathrm{PBN}$ adducts observed following reperfusion in groups I and II is due to continued production of free radicals rather than to slow removal of adducts accumulated during the early phase of reflow.

Group IV $(n=11)$ : control group for EPR signals. As shown in Table II, in the 11 control dogs the 40-min infusion of PBN did not produce any appreciable effect on regional contractile function or hemodynamic variables with the exception of a modest $(25 \pm 10 \%)$ increase in coronary blood flow. Despite the fact that in this group the infusion of PBN was longer (40 $\mathrm{min})$ than in group I (30 $\mathrm{min})$, II (10 min), or III (10 min), no EPR signal was detected in any of the coronary venous blood samples obtained at 5-min intervals throughout the administration of the spin trap. The consistent absence of spin adduct release, coupled with the lack of deterioration in regional function (a parameter which is very sensitive to adverse influences), indicates that the production of adducts in groups I, II, and III was not due to nonspecific toxic effects of PBN.

Effect of PBN on recovery of contractile function. Having observed that PBN traps free radicals in the stunned myocardium, we sought to determine whether this action results in attenuation of postischemic dysfunction. To this end, the dogs in group I (PBN given before ischemia) and group II (PBN given at reperfusion) were compared to control dogs (group V, $n=8$ ) that did not receive PBN. Rather than performing repeated pairwise comparisons among multiple groups at multiple times, we used analysis of variance to assess whether the overall postischemic recovery was modified by $\mathrm{PBN}$, given either before ischemia or at reperfusion (31).

Baseline systolic thickening fraction in the region to be rendered ischemic did not differ among group I (20 $\pm 4 \%)$,

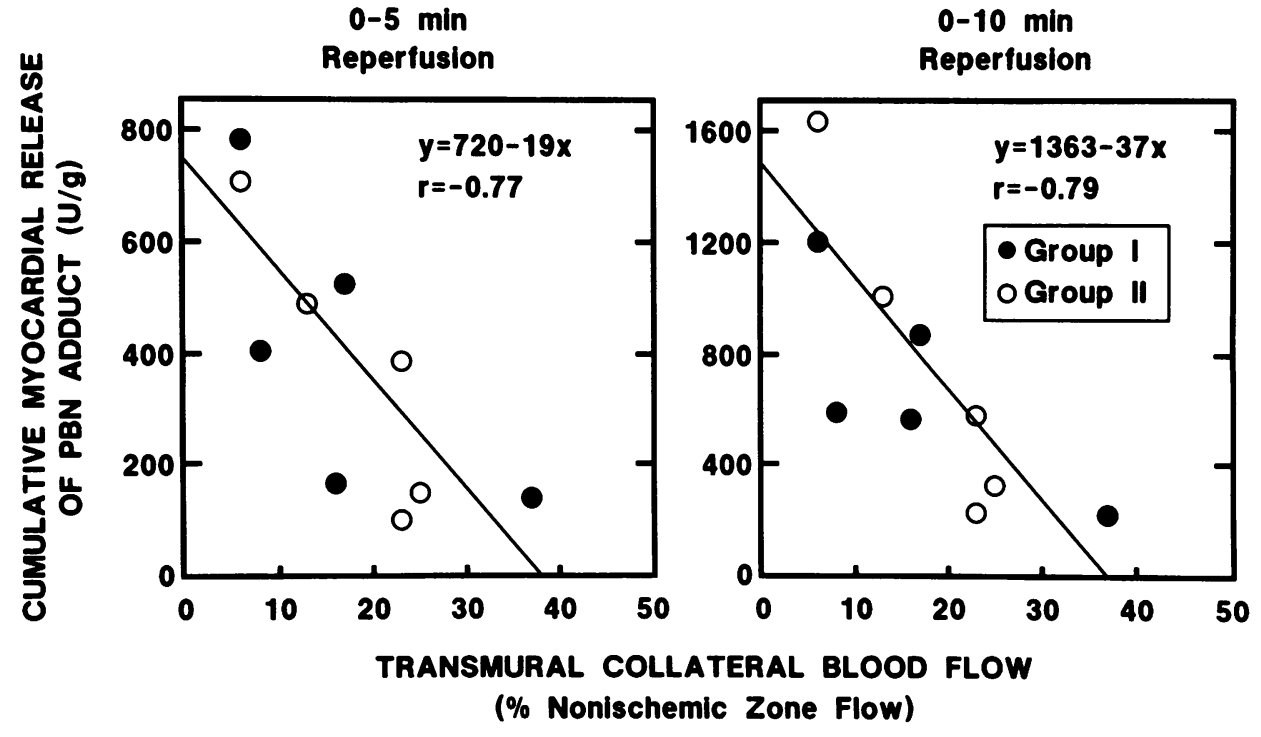

Figure 4. Relationship between mean transmural collateral blood flow to the ischemic region during coronary occlusion (horizontal axis) and total cumulative myocardial release of PBN adducts during the first $5 \mathrm{~min}$ (left) and $10 \mathrm{~min}$ (right) of reperfusion. Collateral flow is expressed as percentage of simultaneous nonischemic zone flow; adduct release is expressed in arbitrary units per gram of myocardium (U/g) (see text for explanation of units). (๑) Dogs in group I (PBN given before ischemia); ( 0 ) dogs in group II (PBN given at reperfusion). In both groups, the myocardial production of PBN adducts after coronary reperfusion was linearly and inversely related to collateral flow during the antecedent occlusion. The regression equations and $r$ values indicated in the figure were obtained by analyzing groups I and II together. 
Table II. Hemodynamic Variables, Wall Thickening, Regional Myocardial Blood Flow, and Occluded Bed Size in Group IV*

\begin{tabular}{|c|c|c|c|}
\hline & \multirow[b]{2}{*}{ Baseline } & \multicolumn{2}{|c|}{ PBN infusion $(\min )$} \\
\hline & & 15 & 40 \\
\hline Heart rate (beats/min) & $150 \pm 6$ & $159 \pm 5$ & $154 \pm 7$ \\
\hline \multicolumn{4}{|l|}{ Mean arterial pressure } \\
\hline$(m m H g)$ & $125 \pm 6$ & $132 \pm 6$ & $134 \pm 8$ \\
\hline \multicolumn{4}{|l|}{ Mean left atrial pressure } \\
\hline$(\mathrm{mmHg})$ & $3 \pm 3$ & $3 \pm 1$ & $3 \pm 1$ \\
\hline \multicolumn{4}{|l|}{ Coronary blood flow } \\
\hline$(\mathrm{ml} / \mathrm{min})^{\ddagger}$ & $22 \pm 5$ & $28 \pm 7$ & $28 \pm 6^{8}$ \\
\hline $\mathrm{LV} \mathrm{d} P / \mathrm{d} t_{\max }(m m H g / s)^{\S}$ & $2,771 \pm 382$ & $2,918 \pm 320$ & $2,857 \pm 298$ \\
\hline $\mathrm{LV} \mathrm{d} P / \mathrm{d} t_{\min }(m m H g / s)^{\|}$ & $3,783 \pm 654$ & $3,796 \pm 803$ & $3,603 \pm 520$ \\
\hline \multicolumn{4}{|l|}{ Thickening fraction (\%) } \\
\hline Posterior LV wall' & $18 \pm 2$ & $18 \pm 3$ & $18 \pm 3$ \\
\hline Anterior LV wall & $15 \pm 2$ & $16 \pm 2$ & $15 \pm 2$ \\
\hline \multicolumn{4}{|l|}{$\begin{array}{l}\text { Transmural regional } \\
\text { myocardial blood } \\
\text { flow }(\mathrm{ml} / \mathrm{min} \text { per } \mathrm{g})\end{array}$} \\
\hline Posterior LV wall & $1.08 \pm 0.16$ & - & $1.32 \pm 0.14^{* *}$ \\
\hline Anterior LV wall & $1.08 \pm 0.13$ & - & $1.18 \pm 0.21$ \\
\hline Occluded vascular bed $(g)$ & \multicolumn{3}{|c|}{$26.9 \pm 2.5 \mathrm{~g}(29.2 \pm 3.1 \%$ of $\mathrm{LV}$ weight $)$} \\
\hline
\end{tabular}

Data are mean \pm SEM.

* In this group PBN was infused for $\mathbf{4 0}$ min but no coronary occlusion was performed.

${ }^{\ddagger}$ Measured in the artery infused with PBN.

${ }^{8} \mathrm{LV} \mathrm{d} P / \mathrm{d} t_{\max }$, maximal rate of left ventricular pressure rise.

" $\mathrm{LV} \mathrm{d} P / \mathrm{d} t_{\min }$, maximal rate of left ventricular pressure fall.

' Vascular bed receiving the infusion of PBN.

** $P<0.05$ vs. baseline.

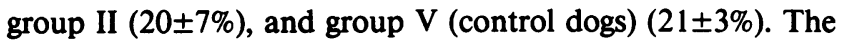
three groups were also similar with respect to the extent of paradoxical systolic thinning (dyskinesis) during ischemia (Fig. 2). After reperfusion, control dogs exhibited minimal recovery of contractile function, and $3 \mathrm{~h}$ after restoration of flow the previously ischemic region was still dyskinetic, indicating severe myocardial stunning. In the dogs receiving PBN, however, recovery of function was greater throughout the 3-h observation period (Fig. 2). Despite the fact that in group II PBN was not given during ischemia, analysis of variance showed that the systolic thickening fraction increased significantly $(P$ $<0.005$ ) after reflow in both group I and II. In contrast, there was no demonstrable change in wall thickening during the occlusion and the reperfusion phase in group $\mathrm{V}(P=0.31)$.

To better assess the effect of PBN using a larger sample size, the dogs in groups I $(n=5)$ and II $(n=5)$ were pooled in one group. The rationale for this was twofold: $(a)$ the ability of PBN to trap free radicals during the burst that followed reperfusion was similar in groups I and II (Fig. 3); (b) there was no appreciable difference between groups I and II with respect to hemodynamics, collateral flow, or occluded bed size (Table I). Again, when the pooled PBN group was compared with the control group, analysis of variance demonstrated that there was a significant $(P<0.05)$ overall intergroup difference in systolic thickening fraction during the reperfusion phase, thus confirming that PBN improved the global pattern of postischemic recovery in the stunned myocardium.

PBN and control groups were similar with respect to sys- temic hemodynamics, collateral flow during coronary occlusion, coronary flow after reperfusion, and occluded bed size (Table I). Thus, the attenuation of stunning by PBN cannot be ascribed to the nonspecific influence of these variables.

\section{Discussion}

This study demonstrates that a brief episode of regional ischemia and the subsequent reperfusion are associated with prolonged free radical generation. The production of free radicals starts within few minutes of coronary occlusion, but most of it takes place in the early phase after restoration of flow, with a burst beginning within seconds of reperfusion. After this initial burst, the production of radicals abates but does not cease, persisting up to $3 \mathrm{~h}$ after reflow. In general, the magnitude of radical generation is inversely related to the adequacy of collateral perfusion, in analogy to the inverse relation previously observed between collateral perfusion and degree of postischemic contractile dysfunction (21). These results provide direct evidence supporting the hypothesis that oxygen metabolites and their reactive secondary products play a significant role in the pathogenesis of the persistent myocardial dysfunction observed after reversible ischemia (myocardial stunning). Previous investigations (15-18) have documented radical generation in isolated, buffer-perfused, nonworking hearts subjected to global ischemia. However, to our knowledge, this is the first study demonstrating production of free radicals in myocardium reperfused after regional ischemia in the intact animal.

Previous studies. Zweier et al. (15) used direct EPR spectroscopy to measure free radical generation in isolated rabbit hearts undergoing $10 \mathrm{~min}$ of global ischemia followed by reperfusion. They observed that the concentration of oxygencentered radicals in the myocardium increased threefold above control during ischemia and sixfold immediately after reperfusion. Garlick et al. (16) studied rat hearts perfused in the Langendorff mode with a buffer containing $3 \mathrm{mM}$ PBN and noted that reperfusion after $15 \mathrm{~min}$ of global ischemia resulted in a burst of PBN radical adduct formation. Two recent reports from Weglicki's laboratory $(17,18)$ describe the appearance of spin adducts of 5,5-dimethyl-1-pyrroline- $N$-oxide in the coronary effluent of isolated rat hearts subjected to periods of global ischemia ranging from 20 to $40 \mathrm{~min}$ followed by reperfusion. In all of the studies using spin traps (16-18), the EPR signals observed were suggestive of both carbon- and oxygen-centered radical adducts.

Although these in vitro observations (15-18) have provided direct evidence that cardiac ischemia and reperfusion are associated with free radical generation, they cannot necessarily be extrapolated to the setting of the stunned myocardium for several reasons. First, the working heart perfused with blood and subjected to regional ischemia in vivo differs in many important respects from the nonworking heart perfused with artificial solutions and subjected to global ischemia in vitro. Secondly, the models used in the aforementioned experiments have not been shown to be associated with stunned myocardium. Myocardial stunning is defined as a persistent but ultimately reversible depression of contractility (3). To determine whether the dysfunction observed in Langendorff preparations does indeed represent stunning, it would be necessary to demonstrate that the postischemic contractile abnormalities are completely reversible; however, the spontaneous 
deterioration of these preparations does not allow such a follow-up.

Two brief reports $(32,33)$ have addressed free radical production during in vivo ischemia. Without using spin traps, Rao et al. (32) detected increased EPR signals in myocardial tissue and local venous effluent blood obtained after $15 \mathrm{~min}$ of ischemia in open-chest dogs. However, these observations were inconclusive because there is no convincing evidence that the paramagnetic species observed were reactive free radicals. The fact that the EPR signal was noted in the venous blood implies that the component responsible for the signal had a stability which would not be characteristic of a reactive free radical. Furthermore, signals due to transition metal complexes were not ruled out and adequate control experiments were not reported.

A recent communication by Arroyo et al. (33) describes results obtained with intracoronary infusion of the spin traps, PBN and 2-methyl-2-nitroso propane (MNP), in dogs undergoing coronary occlusions lasting 20-90 min. At the end of the occlusion, biopsies of ischemic myocardium were obtained and were found to contain spin adducts suggestive of lipid radicals. In this study, the effect of reperfusion was not examined. The preparation employed also differs from ours in that coronary occlusions $\geq 20 \mathrm{~min}$ are associated with variable degrees of irreversible myocardial injury (22). Further, the production of radicals was not assessed in blood-perfused myocardium (the coronary artery was perfused with a buffer solution). The observations of Arroyo and associates are difficult to interpret because no control experiments were reported. It is pertinent to point out that the concentration of spin traps in the coronary perfusate was quite high $(50 \mathrm{mM}$ for both PBN and 2-methyl-2-nitroso propane). We used much lower concentrations of PBN (1.6 mM) because in pilot studies we found that intracoronary infusions of $\mathrm{PBN}$ resulting in estimated coronary blood levels $>20 \mathrm{mM}$ (i.e., $>3.2 \mathrm{mg} / \mathrm{ml}$ ) caused profound toxicity, manifested by rapid, complete, and persistent loss of contractile function (dyskinesis) in the absence of ischemia/reperfusion. Thus, it is difficult to be certain whether the paramagnetic species observed by Arroyo et al. (33) were produced by ischemia or by nonspecific toxic effects of the spin traps on the myocardium.

In summary, the present study differs from previous investigations in that it demonstrates production of free radicals after reversible regional ischemia in the intact animal, it defines the time course of this phenomenon, and it characterizes the relation of radical generation to the severity of the ischemic insult.

Nature of the PBN adducts observed. The spin-trapped radicals described in our report appear to have the same spectra under all the conditions which were investigated (Fig. 1). Analysis of the coupling constants suggests that the signals are due to a mixture of oxygen- and carbon-centered radicals, since (with chloroform as the solvent) the values of $a_{\beta}^{\mathrm{H}}(2.67-2.79 \mathrm{G})$ are smaller than those generally observed with carbon-centered radical adducts of $\mathrm{PBN}(>3.0 \mathrm{G})$, whereas the $a_{\mathrm{N}}$ values (14.75-15.00 G) are larger than those characteristic of oxygen-centered radical adducts of PBN (<14.50 G) (26). In addition to the intermediate values of the coupling constants, the asymmetry of the central doublets (Fig. 1) implies the presence of at least two radical adducts with different $g$ values. Since these adducts are soluble in nonaqueous solvents and resemble the lipid radical adducts of PBN observed in other systems
(26), they may be derived from membrane lipids. However, definitive identification of the adducts will require a substantial investigative effort.

Correlation between severity of ischemia and radical production. No data are available regarding the factors that determine the rate of radical reactions in cardiac ischemia. The present study demonstrates a striking variability in the production of PBN adducts and identifies ischemic perfusion as a major factor responsible for such variability (Fig. 4). The finding that the amount of radicals produced after reflow is determined by the severity of the antecedent ischemia has considerable interest. It has been proposed (34) that the perturbations occurring during ischemia "prime" the myocardium for production of free radicals upon reperfusion. Such perturbations may include the accumulation of reducing equivalents, the disruption of the electron transport chain, the conversion of xanthine dehydrogenase to xanthine oxidase, the activation of the arachidonate cascade, the increased concentration of catecholamines, and the activation of chemotactic mechanisms for neutrophils $(34,35)$. Our study provides evidence supporting this view. The inverse relation between collateral flow during coronary occlusion and radical production after subsequent reperfusion suggests that $(a)$ the mechanisms responsible for the abnormal metabolism of oxygen are activated by ischemia, and $(b)$ the intensity of such activation is determined by the intensity of flow reduction. In a sense, it appears that the amount of free radicals that will be produced by the heart after reperfusion (and the consequent cell damage) are already "predetermined," at least in part, before flow is restored. These findings also suggest that interventions which improve perfusion during ischemia might attenuate free radical reactions following reflow.

Time course of PBN adduct production. The finding that the burst of spin adduct formation peaked 2-4 min after reperfusion (Fig. 3) implies that before this, there is a burst of free radical production in the myocardium. Our data are therefore consistent with the concept that free radical generation increases abruptly very early (within moments) after reflow, in accordance with in vitro studies (15-17). However, an interesting (and somewhat unexpected) finding was that, although the myocardial release of spin adducts was most intense during the initial minutes of reperfusion, it continued for a relatively long time thereafter $(3 \mathrm{~h})$. Zweier et al. (15) found that in isolated hearts the concentration of oxygen-centered radicals peaked $10 \mathrm{~s}$ after reflow and was still elevated at $60 \mathrm{~s}$, but no observation was made thereafter. In in vitro experiments employing 3 $\mathrm{mM}$ PBN (16) and $65 \mathrm{mM}$ 5,5-dimethyl-1-pyrroline- $N$-oxide (17), the production of spin adducts was maximal 3-4 min after restoration of coronary flow and ceased within the subsequent $10 \mathrm{~min}$. The reason for the different duration of spin adduct production in these studies vs. the present investigation is not clear, but it may relate to dissimilarities in the extraction techniques, in the sensitivity of the EPR analyses, and/or in the experimental conditions employed (in vitro vs. in vivo). As mentioned above, the EPR signals detected in our study appear to be due to a combination of oxygen- and carbon-centered radicals. Conceivably, both of these adducts may be produced during oxy-radical-initiated lipid peroxidation, since this process gives rise to alkyl as well as alkoxy radicals (34), both of which can be trapped by PBN (26). Thus, as has been suggested (34), the generation of oxy-radicals may be in itself a relatively brief event, but these species could initiate a self- 
propagating process of lipid peroxidation that could continue in the membrane lipids even after the production of the initiating radical species has ceased.

Attenuation of myocardial stunning by PBN. The finding that PBN enhanced postischemic recovery of function further corroborates the hypothesis that free radical species contribute to myocardial stunning. Like other spin traps, PBN in effect acts as a free radical scavenger (19). Accordingly, it seems likely that the beneficial effect of this agent on contractility were due to removal of injurious radical species.

PBN afforded only partial protection against postischemic dysfunction. However, it is important to keep in mind that the influence of this agent on the stunned myocardium depends on the collective effectiveness of the substances with which it is competing and on the configuration of the dose-response curve-two variables which are basically unknown. It is possible that our dose of PBN trapped only a relatively small fraction of the total number of radicals produced. On the other hand, the radicals that we have observed are probably secondary species (e.g., alkyl and/or alkoxy radicals) formed in the course of lipid peroxidation reactions initiated by the primary oxygen radicals (see above). Trapping of secondary radicals by PBN might afford incomplete protection if the critical damage is caused mainly by the primary radicals.

Methodological implications. The present study has also important methodological implications. Previous experiments have shown that PBN and 5,5-dimethyl-1-pyrroline- $N$-oxide can detect radical generation after cardiac ischemia in vitro (16-18). Others have demonstrated that PBN can effectively trap the radicals formed in vivo by $\gamma$ radiation (28) and by metabolism of halothane (25), $\mathrm{CCl}_{4}$ (26), and ethanol (27). However, the technique of administering spin traps in vivo and performing serial analyses of spin adducts in the coronary effluent has not been previously described. By demonstrating the usefulness of applying spin trapping to the investigation of myocardial ischemia in the intact animal, our results significantly expand the possible applications of the method. The new approach described herein has the potential not only to characterize radical generation in vivo, but also to delineate its time course and to detect relative changes in its intensity; thus, it may be used to quantitatively assess the effects of antioxidant interventions. The technique appears to be more suitable than direct EPR spectroscopy for studies in intact animals. Unlike direct EPR measurements (which can be performed at one time point only), spin trapping can provide an integrated measure of radical generation over a given interval of time (19). This is particularly important in view of the prolonged duration of free radical production in the stunned myocardium (Fig. 3). Further, spin trapping is applicable to the study of ischemia/reperfusion in various organs and, ultimately, even in conscious animal preparations, which is not possible with direct EPR spectroscopy.

Conclusions. In conclusion, a major limitation of the hypothesis that oxygen metabolites mediate myocardial stunning has been the circumstantial nature of the evidence available. By demonstrating that brief ischemia is associated with production of free radicals in vivo, our study provides significant new evidence to support this hypothesis. After a phase of indirect investigative approaches, it is now important to test the free radical theory of ischemia/reperfusion injury by using more direct methods designed to detect and measure free radi- cals in preparations that are as physiological as possible. The technique described in this study should be a useful step in this direction.

\section{Acknowledgments}

We thank Dr. Coit M. DuBose, for helpful discussion, Jennifer Pocius and Curtis Mills for excellent technical assistance, and Bonnie Anderson for expert secretarial assistance.

This study was supported in part by grant-in-aid 87R-171 from the American Heart Association, Texas Affiliate, grant GM-36512 from the National Institutes of Health, Biomedical Research Support grant 2507RR05538-25, and grants HL-36277 and HL-23161 from the National Heart, Lung, and Blood Institute. Dr. Bolli is a recipient of the Physician-Scientist Award of the American College of Chest Physicians.

\section{References}

1. Heyndrickx, G. R., R. W. Millard, R. J. McRitchie, P. R. Maroko, and S. F. Vatner. 1975. Regional myocardial functional and electrophysiological alterations after brief coronary artery occlusion in conscious dogs. J. Clin. Invest. 56:978-985.

2. Kloner, R. A., S. G. Ellis, R. Lange, and E. Braunwald. 1983. Studies of experimental coronary artery reperfusion: effects on infarct size, myocardial function, biochemistry, ultrastructure and microvascular damage. Circulation. 68(Suppl. I):I-8-I-15.

3. Braunwald, E., and R. A. Kloner. 1982. The stunned myocardium: Prolonged, postischemic ventricular dysfunction. Circulation. 66:1146-1149.

4. Ciuffo, A. A., P. Ouyant, L. C. Becker, L. Levin, and M. L. Weisfeldt. 1985. Reduction of sympathetic inotropic response after ischemia in dogs. J. Clin. Invest. 75:1504-1509.

5. Jennings, R. B., J. Schaper, M. L. Hill, C. Steenbergen, Jr., and K. A. Reimer. 1985. Effect of reperfusion late in the phase of reversible ischemic injury: changes in cell volume, electrolytes, metabolites, and ultrastructure. Circ. Res. 56:262-278.

6. Reimer, K. A., M. L. Hill, and R. B. Jennings. 1981. Prolonged depletion of the adenine nucleotide pool due to delayed resynthesis of adenine nucleotides following reversible myocardial ischemic injury in dogs. J. Mol. Cell. Cardiol. 13:229-239.

7. Myers, M. L., R. Bolli, R. F. Lekich, C. J. Hartley, and R Roberts. 1985. Enhancement of recovery of myocardial function by oxygen free-radical scavengers after reversible regional ischemia. $\mathrm{Cir}$ culation. 72:915-921.

8. Gross, G. J., N. E. Farber, H. F. Hardman, and D. C. Warltier. 1986. Beneficial actions of superoxide dismutase and catalase in stunned myocardium of dogs. Am. J. Physiol. 250:H372-H377.

9. Przyklenk, K., and R. A. Kloner. 1986. Superoxide dismutase plus catalase improve contractile function in the canine model of the "stunned" myocardium. Circ. Res. 58:148-156.

10. Myers, M. L., R. Bolli, R. F. Lekich, C, J. Hartley, and R. Roberts. 1986. N-2-mercaptopropionylglycine improves recovery of myocardial function after reversible regional ischemia. J. Am. Coll. Cardiol. 8:1161-1168.

11. Bolli, R., W. X. Zhu, C. J. Hartley, L. H. Michael, J. Repine, M. L. Hess, R. C. Kukreja, and R. Roberts. 1987. Dimethylthiourea attenuates dysfunction in the postischemic "stunned" myocardium. Circulation. 76:458-468.

12. Charlat, M. L., P. G. O'Neill, J. M. Egan, D. R. Abernethy, L. H. Michael, M. L. Myers, R. Roberts, and R. Bolli. 1987. Evidence for a pathogenetic role of xanthine oxidase in the "stunned" myocardium. Am. J. Physiol. 252:H566-H577.

13. Puett, D. W., M. B. Forman, C. U. Cates, B. H. Wilson, K. R. Hande, G. C. Friesinger, and R. Virmani. 1987. Oxypurinol limits 
myocardial stunning but does not reduce infarct size after reperfusion. Circulation. 76:678-686.

14. Bolli, R., B. S. Patel, W. X. Zhu, P. G. O'Neill, M. L. Charlat, and R. Roberts. 1987. The iron chelator desferrioxamine attenuates postischemic ventricular dysfunction. Am. J. Physiol. 253:H1372H1380.

15. Zweier, J. L., J. T. Flaherty, and M. L. Weisfeldt. 1987. Direct measurement of free radical generation following reperfusion of ischemic myocardium. Proc. Natl. Acad. Sci. USA. 84:1404-1407.

16. Garlick, P. B., M. J. Davies, D. J. Hearse, and T. F. Slater. 1987. Direct detection of free radicals in the reperfused rat heart using electron spin resonance spectroscopy. Circ. Res. 61:757-760.

17. Kramer J. H., C. M. Arroyo, B. F. Dickens, and W. B. Weglicki. 1987. Spin-trapping evidence that graded myocardial ischemia alters post-ischemic superoxide production. Free Radical Biol. Med. 3:153159.

18. Arroyo, C. M., J. H. Kramer, B. F. Dickens, and W. B. Weglicki. 1987. Identification of free radicals in myocardial ischemia/reperfusion by spin trapping with nitrone DMPO. FEBS (Fed. Eur. Biochem. Soc.) Lett. 221:101-104.

19. Rosen G. M., and E. Finkelstein. 1985. Use of spin traps in biological systems. Adv. Free Radical Biol. Med. 1:347-375.

20. Zhu, W. X., M. L. Myers, C. J. Hartley, R. Roberts, and R. Bolli. 1986. Validation of a single crystal for measurement of transmural and epicardial thickening. Am. J. Physiol. 251:H1045-H1055.

21. Bolli, R., W. X. Zhu, J. I. Thornby, P. G. O'Neill, and R. Roberts. 1988. Time-course and determinants of recovery of function after reversible ischemia in conscious dogs. Am. J. Physiol. 254:H102-H114.

22. Jennings, R. B., and K. A. Reimer. 1983. Factors involved in salvaging ischemic myocardium: effects of reperfusion of arterial blood. Circulation. 68(Suppl. I):I-25-I-36.

23. Lie, J. T., P. C. Pairolero, K. E. Holley, and J. L. Titus. 1975. Macroscopic enzyme-mapping verification of large, homogenous, experimental myocardial infarcts of predictable size and location in dogs. J. Thorac. Cardiovasc. Surg. 60:599-605.

24. Hearse, D. J., and A. Tosaki. 1987. Free radicals and reperfu- sion-induced arrhythmias: protection by spin trap agent PBN in the rat heart. Circ. Res. 60:375-383.

25. Poyer, J. L., P. B. McCay, C. C. Weddle, and P. E. Downs. 1981. In vivo spin-trapping of radicals formed during halothane metabolism. Biochem. Pharmacol. 30:1517-1519.

26. McCay, P. B., E. K. Lai, J. L. Poyer, C. M. DuBose, and E. G. Janzen. 1984. Oxygen- and carbon-centered free radical formation during carbon tetrachloride metabolism. J. Biol. Chem. 259:21352142.

27. Reinke, L. A., E. K. Lai, C. M. DuBose, and P. B. McCay. 1987. Reactive free radical generation in vivo in the heart and liver of ethanol-fed rats: correlation with in vitro radical formation. Proc. Natl. Acad. Sci. USA. 84:9223-9227.

28. Lai, E. K., C. Crossley, R. Sridhar, H. P. Misra, E. G. Janzen, and P. B. McCay. 1986. In vivo spin trapping of free radicals generated in brain, spleen, and liver during gamma radiation of mice. Arch. Biochem. Biophys. 244:156-160.

29. Hartley, C. J., L. A. Latson, L. H. Michael, C. L. Seidel, R. M. Lewis, and M. L. Entman. 1983. Doppler measurement of myocardial thickening with a single epicardial transducer. Am. J. Physiol. 245:H1066-H1072.

30. Roan, P., F. Scales, S. Saffer, L. M. Buja, and J. T. Willerson. 1979. Functional characterization of left ventricular segmental responses during the initial 24 hours and first week after experimental canine myocardial infarction. J. Clin. Invest. 64:1074-1088.

31. Armitage, P. 1977. Statistical Methods in Medical Research. Blackwell Scientific Publications, Oxford. 189-216.

32. Rao, P. S., M. V. Cohen, and H. S. Mueller. 1983. Production of free radicals and lipid peroxides in early experimental myocardial ischemia. J. Mol. Cell. Cardiol. 15:713-716.

33. Arroyo, C. M., J. H. Kramer, R. H. Leiboff, G. W. Mergner, B. F. Dickens, and W. B. Weglicki. 1987. Spin trapping of oxygen and carbon-centered free radicals in ischemic canine myocardium. Free Radical Biol. Med. 3:313-316.

34. Thompson, J. A., and M. L. Hess. 1986. The oxygen free radical system: a fundamental mechanism in the production of myocardial necrosis. Prog. Cardiovasc. Dis. 28:449-462.

35. McCord, J. M. 1985. Oxygen-derived free radicals in postischemic tissue injury. N, Engl. J. Med. 312:159-163. 